\title{
Morphological Variations among Different Jackfruit Genotypes
}

\author{
Pebbuli Avani* and F.K. Bauri \\ Department of Fruits Science, BCKV, Mohanpur 741252, West Bengal, India \\ *Corresponding author
}

\section{A B S T R A C T}

\section{Keywords \\ Plant characters, Leaf characters and PCA (Principle component Analysis) \\ Article Info \\ Accepted: \\ 22 October 2018 \\ Available Online: \\ 10 November 2018}

\begin{abstract}
Jackfruit (Artocarpus heterophyllus Lam.), being a cross pollinated crop shows high variations in plant and leaf characters. Thus, present study was under taken to assess the morphological variations of plant and leaf characters among twenty genotypes under AICRP on Fruits, B.C.K.V., Mohanpur centre as per the descriptor developed by Bioversity International (IPGRI). Variations were noticed with respect to shape of crown (elliptic, pyramidal, broadly pyramidal, semicircular and irregular), leaf shape (elliptic, oblong, obovate, narrowly elliptic and broadly elliptic) and also in terms of biometric characters of fruit like plant height $(5.9-9.1 \mathrm{~m})$, trunk circumference $(0.61-1.17 \mathrm{~m})$, crown diameter $(5.1-10.2 \mathrm{~m})$, leaf blade length $(7.5-13.4 \mathrm{~cm})$, leaf blade diameter $(4.1-7.6 \mathrm{~cm})$, petiole length $(0.7-2.7 \mathrm{~cm})$.
\end{abstract}

\section{Introduction}

Jackfruit (Artocarpus heterophyllus Lam.), is a tetraploid $(4 n=56)$ and is the one of the most significant trees in tropical homegardens and perhaps the most widespread and useful tree in the important genus Artocarpus, of family Moraceae. The jackfruit species reportedly originated in the rainforests of the Western Ghats of India (Chandler, 1958) and in Malaysia (Brown, 1941). Jackfruit is a national fruit of Bangladesh and state fruit of Kerala and Tamilnadu; where it is commonly referred to as "poor man's food" as it is cheap and plentiful during the season. In India, it has wide distribution in Assam, Tripura, Bihar, Uttar Pradesh, the foothills of the Himalayas and South Indian States of Kerala, Tamil Nadu and Karnataka.
Jackfruit is a multipurpose tree and all parts of the plant are equally important. Fruits are rich in several nutrients. It can act as source of complete nutrition to the consumer. Fruits of jackfruit are compared to avocado and olive in terms of the healthier mix of nutrients for human dietary needs, almost having the exact nutrient equivalent of mother's milk. It is rich in vit- $\mathrm{B}$ and $\mathrm{C}$, potassium, calcium, iron, proteins and high level of carbohydrates, affordable and readily available supplement to our staple food. Its seeds are rich in proteins and can be relished as a nutritious nut. The fruit is also the source of chemical 'Jacalin' useful in preventing colon cancer, AIDS (Priya et al., 2014). Tree is known for its durable anti-termite timber. Jackfruit trees are suitable for growing in wide agro-climatic regions owing to its versatile adaptability, 
hardy nature, low maintenance cost and high yield. The trees helps in amelioration of soils and prevention of the soil erosion. The leaves and fruit waste provide valuable fodder for cattle, pigs and goats.

Jackfruit, being cross-pollinated and mostly seed propagated, jackfruit has innumerable types or forms in terms of fruit characteristics. The types differ widely among themselves and many types available in India under various local names have originated in this way. However, distinct cultivars are not available. Selection of superior genotypes for the fresh market and the processing industry, as well as for high yield, better quality and tolerance to abiotic and biotic stresses, would be of great value for commercialization of this underutilized nutritive fruit. Thus, present study was under taken to assess the morphological variations among twenty genotypes under AICRP on Fruits, B.C.K.V., Mohanpur centre as per the descriptor developed by Bioversity International (IPGRI)

\section{Materials and Methods}

A study entitled "Morphological variations among different jackfruit genotypes" was conducted at AICRP on Fruits, B.C.K.V., Mohanpur centre during 2015-2017. Various morphological characters o jackfruit plant and leaf were observed in twenty different genotypes (serially numbered as G-1 to G-20) as per the descriptor developed by Bioversity International (IPGRI). The plants were healthy, about 14 years of age and received similar cultural treatments.

Plant characters viz., age of the tree, tree height $(\mathrm{m})$, trunk circumference $(\mathrm{cm})$, trunk surface, trunk growth habit, crown diameter (m) and crown shape were recorded on unpruned plants. Lea characters viz., leaf blade length $(\mathrm{cm})$, leaf blade width $(\mathrm{cm})$, leaf blade shape, leaf apex shape, leaf base shape, leaf blade margin, leaf upper surface pubescence, leaf lower surface pubescence, leaf midrib pubescence, petiole length $(\mathrm{mm})$ and grooves on petiole were recorded by collecting five leaves from each genotype from all sides crown.

Principal components were computed from the correlation matrix and genotypic scores obtained for the first component and succeeding components with latent roots greater than unity (Jager et al., 1983).

\section{Results and Discussion}

All 20 genotypes plants selected for present study were of same age i.e. fourteen (14) years old. Even with the same age of the genotypes, differences with regard to plant height were observed among the genotypes (Table 1). The maximum plant height of $9.1 \mathrm{~m}$ was observed in G-20, followed by G-12 (8.9m) and G-16 $(8.8 \mathrm{~m})$. The least height of $5.9 \mathrm{~m}$ was observed in G-4. Differences in plant height was also observed by Gaithoiliu et al., (2017) and found height of different genotypes ranges from $6.5 \mathrm{~m}$ to $16 \mathrm{~m}$.

The results of trunk circumference varied among the genotypes (Table 1), maximum trunk circumference $(1.17 \mathrm{~m})$ was recorded in G-10. The least trunk circumference $(0.61 \mathrm{~m})$ was observed in G-4. Grand mean of crown diameter recorded was $7.42 \mathrm{~m}$ and showing variation from $5.1 \mathrm{~m}(\mathrm{G}-18)$ to $10.2 \mathrm{~m}(\mathrm{G}-14)$.

Semi-erect plant growth habit recorded in G-3, G-5, G-6, G-7, G-8, G-9, G-10, G-11, G-13, G-14, G-15, G-17, G-18 and G-20 whereas spreading growth habit observed in G-1, G-2, G-4, G-12, G-16 and G-19. Two types of trunk surface (rough and very rough). Among them 16 showed rough surface (G-1, G-3, G-4, G-5, G-6, G-8, G-9, G-11, G-12, G-13, G-14, G-15, G-17, G-18, G-19 and G-20) and 4 recorded very rough $(\mathrm{G}-2, \mathrm{G}-7, \mathrm{G}-10$ and G- 
16). Five crown shapes were noted i.e. elliptic in G-3, G-4, G-5, G-17; pyramidal in G-1, G9, G-11; broadly pyramidal in G-2, G-6; G-8, 10, G-12, G-13, G-14, G-15, G-16, G-20; semicircular in G-7 and irregular in G-18 and G-19. Terminal (2012) also reported the varied plant crown shape like irregular, oblong, pyramidal, obovate, semicircular, elliptical and broadly pyramidal in both clonal and seedling progenies.

The genotypes differed in leaf length and ranged from $7.5 \mathrm{~cm}$ to $13.4 \mathrm{~cm}$ for the various jackfruit genotypes studied (Table 2). The grand mean leaf length for the genotypes studied was $10.6 \mathrm{~cm}$. The maximum leaf length of $13.40 \mathrm{~cm}$ was recorded in genotypes G-13 and G-15 followed by G-11 $(12.00 \mathrm{~cm})$, while lowest leaf length $(7.5 \mathrm{~cm})$ was recorded in G-10. Grand mean leaf width was observed to be $5.7 \mathrm{~cm}$ for the jackfruit genotypes studied, while the leaf width ranged from 4.1 $\mathrm{cm}$ to $7.6 \mathrm{~cm}$. The maximum leaf width of 7.6 $\mathrm{cm}$ was recorded in G-14, while lowest leaf width of $4.1 \mathrm{~cm}$ was recorded in G-20. Variation in leaf blade length and width was also reported in the study of Ruby Khan et al., (2010). Five different leaf blade shapes were noted as elliptic in G-1, G-4, G-6, G-9, G-18, G-19; oblong in G-8, G-12, G-14, G-17; obovate in G-3, G-7; narrowly elliptic in G-5, G-11, G-13, G-15, G-16, G-20 and broadly elliptic in G-2 and G-10. Similar observations in conformity to this finding were also reported by Chandan (2001) with elliptic, narrowly elliptic, broadly elliptic, obovate, oblong and lyrate. The shape of the leaf apex were noted as acute (G-2, G-6, G-7, G-11, G13, G-16 and G-20), acuminate (G-1, G-3, G4, G-5, G-9, G-15, G-18 and G-19) and obtuse (G-8, G-10, G-12, G-14 and G-17). Whereas, four different shape of the leaf apex were noted as cuneate (G-2, G-3, G-5, G-11, G-13, G-14, G-15, G-16, G-19 and G-20), shortly attenuate (G-1, G-7, G-9, G-10, G-12, G-17 and G-18), oblique (G-4 and G-6) and rounded
(G-8). Variation in leaf base shape and apex shape were also reported in the study of Chandrasekhar (2014). The leaf margin observed was undulated (G-1, G-2, G-3, G-4, G-5, G-7, G-8, G10, G-12, G-13, G-14, G-15, G-17, G-18 and G-19) and entire (G-6, G-9, G-11, G-16 and G-20). From the data represented in Table 2 revealed that leaf upper surface pubescence showing both sparse $(\mathrm{G}-1$, G-2, G-3, G-4, G-5, G-8, G-10, G-11, G-12, G-13, G-14, G-15, G-17, G-18, G-19) and glabrous (G-6, G-7, G-9, G-16 and G-20).

Leaf lower surface pubescence also showed both sparse (G-1, G-2, G-3, G-4, G-5, G-7, G8, G-9, G-10, G-11, G-12, G-13, G-14, G-15, G-17, G-18, G-19) and glabrous (G-6, G-16 and G-20). Gaithoiliu et al., (2017) also reported varied intensity of leaf upper and surface pubescence. leaf midrib pubescence showing sparsely pubescence (G-1, G-2, G-3, G-4, G-5, G-7, G-8, G-10, G-11, G-12, G-13, G-14, G-15, G-17, G-18, G-19) and glabrous (G-6, G-16 and G-20). The genotypes differed in petiole length and the average petiole length ranged from $0.7 \mathrm{~cm}$ to $2.70 \mathrm{~cm}$ for the various jackfruit genotypes studied. The grand mean petiole length for the genotypes studied was $1.7 \mathrm{~cm}$. Maximum petiole length of $2.70 \mathrm{~cm}$ was recorded in genotype G-14 followed by G-19 $(2.20 \mathrm{~cm})$ and G-15 $(2.20 \mathrm{~cm})$, while lowest petiole length of $0.7 \mathrm{~cm}$ was recorded in G-5. Variation in leaf blade length was also reported in the study of Wangchu (2005) ranging $0.08 \mathrm{~cm} \quad(\mathrm{~T}-4)$ to $2.50 \mathrm{~cm} \quad(\mathrm{~T}-27)$. Presence of groove, acute crotch angle and rounded leaf petiole shape was recorded in all twenty genotypes.

An overall perusal of results revealed that medium plant height genotypes G-2 (7.8m), G-5 (7.5m) and G-18 (6.0m) with higher number of fruits per plant was noted 27, 68 and 30 fruits respectively, which gives opportunity to grow as homestead plants with good fruit quality fruits. 
Table.1 Plant characters of different selected jackfruit genotypes

\begin{tabular}{|c|c|c|c|c|c|c|c|c|c|c|}
\hline Genotype & $\begin{array}{c}\text { Plant Heigh } \\
{[\mathrm{m}]}\end{array}$ & $\begin{array}{c}\text { Trunk } \\
\text { Circumference } \\
{[\mathrm{m}]}\end{array}$ & $\begin{array}{c}\text { Crown } \\
\text { Diameter [m] } \\
\mathbf{N}-\mathbf{S}\end{array}$ & $\begin{array}{c}\text { Crown } \\
\text { Diameter } \\
{[\mathrm{m}] \mathrm{E}-\mathrm{W}}\end{array}$ & $\begin{array}{l}\text { Mean Crown } \\
\text { Diameter }[\mathrm{m}]\end{array}$ & $\begin{array}{c}\text { Trunk Growth } \\
\text { Habit }\end{array}$ & $\begin{array}{c}\text { Trunk } \\
\text { Surface }\end{array}$ & Crown Shape & $\begin{array}{c}\text { No. of } \\
\text { Fruits/Plant }\end{array}$ & $\begin{array}{l}\text { Fruit } \\
\text { Weight } \\
\text { (Kg) }\end{array}$ \\
\hline G-1 & 7.5 & 0.72 & 6.5 & 6.4 & 6.45 & Spreading & Rough & Pyramidal & 11 & 9 \\
\hline G-2 & 7.8 & 0.68 & 8.2 & 6.2 & 7.2 & Spreading & $\begin{array}{c}\text { Very } \\
\text { Rough }\end{array}$ & Broadly Pyramidal & 27 & 9 \\
\hline G-3 & 7.1 & 0.86 & 6.9 & 7.6 & 7.25 & Semi-Erect & Rough & Elliptical & 12 & 10 \\
\hline G-4 & 5.9 & 0.61 & 5.2 & 5.3 & 5.25 & Spreading & Rough & Elliptical & 9 & 7 \\
\hline G-5 & 7.5 & 0.81 & 8.3 & 8.3 & 8.3 & Semi-Erect & Rough & Elliptical & 68 & 3 \\
\hline G-6 & 8.8 & 0.92 & 9.1 & 8.8 & 8.95 & Spreading & Rough & Broadly Pyramidal & 9 & 7 \\
\hline G-7 & 8.4 & 0.83 & 8.7 & 8.9 & 8.8 & Semi Erect & $\begin{array}{c}\text { Very } \\
\text { Rough }\end{array}$ & Semicircular & 13 & 11 \\
\hline G-8 & 8.6 & 0.89 & 8.9 & 9.1 & 9 & Spreading & Rough & Broadly Pyramidal & 8 & 6 \\
\hline G-9 & 7.5 & 0.70 & 7 & 6.6 & 6.8 & Semi Erect & Rough & Pyramidal & 9 & 7 \\
\hline G-10 & 8.7 & 1.17 & 8.7 & 8.9 & 8.8 & Semi-Erect & $\begin{array}{c}\text { Very } \\
\text { Rough }\end{array}$ & Broadly Pyramidal & 11 & 9 \\
\hline G-11 & 8.2 & 0.81 & 8.1 & 7.6 & 7.85 & Semi Erect & Rough & Pyramidal & 7 & 5 \\
\hline $\mathrm{G}-12$ & 8.9 & 0.91 & 9.3 & 9.7 & 9.5 & Spreading & Rough & Broadly Pyramidal & 9 & 7 \\
\hline G-13 & 7.5 & 0.76 & 7.9 & 7.1 & 7.5 & Semi Erect & Rough & Broadly Pyramidal & 18 & 16 \\
\hline G-14 & 8.1 & 0.89 & 10.7 & 9.7 & 10.2 & Semi-Erect & Rough & Broadly Pyramidal & 9 & 7 \\
\hline$G-15$ & 7.5 & 0.70 & 7 & 6.6 & 6.8 & Semi Erect & Rough & Broadly Pyramidal & 7 & 5 \\
\hline$G-16$ & 8.8 & 1.10 & 9.2 & 8.5 & 8.85 & Spreading & $\begin{array}{c}\text { Very } \\
\text { Rough }\end{array}$ & Broadly Pyramidal & 6 & 5 \\
\hline G-17 & 7.3 & 0.86 & 7.7 & 7.5 & 7.6 & Semi-Erect & Rough & Elliptical & 7 & 5 \\
\hline G-18 & 6 & 0.64 & 5.1 & 5.1 & 5.1 & Semi-Erect & Rough & Irregular & 30 & 5 \\
\hline G-19 & 7.3 & 0.73 & 6.7 & 6.3 & 6.5 & Semi-Erect & Rough & Irregular & 7 & 5 \\
\hline G-20 & 9.1 & 0.93 & 7.9 & 8.4 & 8.15 & Semi-Erect & Rough & Broadly Pyramidal & 8 & 6 \\
\hline General Mean & 7.57 & 0.82 & 7.53 & 7.31 & 7.42 & & & & 14.3 & 7.2 \\
\hline Max. & $9.1(\mathrm{G}-20)$ & $1.17(\mathrm{G}-10)$ & $10.7(\mathrm{G}-14)$ & $\begin{array}{c}9.7(\mathrm{G}-12 \& \\
\mathrm{G}-14)\end{array}$ & $10.2(\mathrm{G}-14)$ & & & $68(\mathrm{G}-1)$ & $\begin{array}{c}16 \\
(\mathrm{G}-13)\end{array}$ & \\
\hline Min. & $5.9(\mathrm{G}-4)$ & $0.61(\mathrm{G}-4)$ & $5.1(\mathrm{G}-18)$ & $5.1(\mathrm{G}-18)$ & $5.1(\mathrm{G}-18)$ & & & $6(\mathrm{G}-16)$ & $3(\mathrm{G}-5)$ & \\
\hline
\end{tabular}


Table.2 Leaf characters of different selected jackfruit genotypes

\begin{tabular}{|c|c|c|c|c|c|c|c|c|c|c|c|}
\hline & notype & $\begin{array}{c}\text { Leaf } \\
\text { Blade } \\
\text { Length } \\
(\mathrm{cm})\end{array}$ & $\begin{array}{c}\text { Leaf } \\
\text { Blade } \\
\text { Width } \\
(\mathrm{cm})\end{array}$ & $\begin{array}{l}\text { Leaf Blade } \\
\text { Shape }\end{array}$ & $\begin{array}{c}\text { Leaf } \\
\text { Apex } \\
\text { Shape }\end{array}$ & $\begin{array}{l}\text { Leaf Base } \\
\text { Shape }\end{array}$ & $\begin{array}{l}\text { Leaf Blade } \\
\text { Margin }\end{array}$ & $\begin{array}{l}\text { Leaf Upper } \\
\text { Surface } \\
\text { Pubescence }\end{array}$ & $\begin{array}{l}\text { Leaf Lower } \\
\text { Surface } \\
\text { Pubescence }\end{array}$ & $\begin{array}{l}\text { Leaf Midrib } \\
\text { Pubescence }\end{array}$ & $\begin{array}{c}\text { Petiole } \\
\text { Length } \\
(\mathbf{C m})\end{array}$ \\
\hline & G-1 & 9.4 & 5.1 & Elliptic & $\begin{array}{l}\text { Acumi } \\
\text { nate }\end{array}$ & $\begin{array}{c}\text { Shortly } \\
\text { Attenuate }\end{array}$ & Undulate & Sparse & Sparse & $\begin{array}{l}\text { Sparsely } \\
\text { Puberulent }\end{array}$ & 1.4 \\
\hline & G-2 & 11.0 & 5.8 & $\begin{array}{l}\text { Broadly } \\
\text { Elliptic }\end{array}$ & Acute & Cuneate & Undulate & Sparse & Sparse & $\begin{array}{c}\text { Sparsely } \\
\text { Puberulent }\end{array}$ & 1.7 \\
\hline & G-3 & 10.9 & 5.9 & Obovate & $\begin{array}{l}\text { Acumi } \\
\text { nate }\end{array}$ & Cuneate & Undulate & Sparse & Sparse & $\begin{array}{c}\text { Sparsely } \\
\text { Puberulent }\end{array}$ & 1.8 \\
\hline & $\mathrm{G}-4$ & 9.6 & 5.0 & Elliptic & $\begin{array}{l}\text { Acumi } \\
\text { nate }\end{array}$ & Oblique & Undulate & Sparse & Sparse & $\begin{array}{c}\text { Sparsely } \\
\text { Puberulent }\end{array}$ & 1.5 \\
\hline & G-5 & 9.5 & 4.7 & $\begin{array}{l}\text { Narrowly } \\
\text { Elliptic }\end{array}$ & $\begin{array}{l}\text { Acumi } \\
\text { nate }\end{array}$ & Cuneate & Undulate & Sparse & Sparse & $\begin{array}{c}\text { Sparsely } \\
\text { Puberulent }\end{array}$ & 0.7 \\
\hline & G-6 & 11.2 & 6.2 & Elliptic & Acute & Oblique & Entire & Glabrous & Glabrous & Glabrous & 2.1 \\
\hline & $\overline{G-7}$ & 8.3 & 5.5 & Obovate & Acute & $\begin{array}{c}\text { Shortly } \\
\text { Attenuate }\end{array}$ & Undulate & Glabrous & Sparse & $\begin{array}{c}\text { Sparsely } \\
\text { Puberulent }\end{array}$ & 1.5 \\
\hline & G-8 & 8.4 & 5.7 & Oblong & Obtuse & Rounded & Undulate & Sparse & Sparse & $\begin{array}{c}\text { Sparsely } \\
\text { Puberulent }\end{array}$ & 0.8 \\
\hline & G-9 & 11.4 & 6.5 & Elliptic & $\begin{array}{l}\text { Acumi } \\
\text { nate }\end{array}$ & $\begin{array}{c}\text { Shortly } \\
\text { Attenuate }\end{array}$ & Entire & Glabrous & Sparse & Glabrous & 1.7 \\
\hline & $\overline{G-10}$ & 7.5 & 5.9 & $\begin{array}{l}\text { Broadly } \\
\text { Elliptic }\end{array}$ & Obtuse & $\begin{array}{c}\text { Shortly } \\
\text { Attenuate }\end{array}$ & Undulate & Sparse & Sparse & $\begin{array}{l}\text { Sparsely } \\
\text { Puberulent }\end{array}$ & 1.3 \\
\hline & $G-11$ & 12.0 & 6.1 & $\begin{array}{l}\text { Narrowly } \\
\text { Elliptic }\end{array}$ & Acute & Cuneate & Entire & Sparse & Sparse & $\begin{array}{c}\text { Sparsely } \\
\text { Puberulent }\end{array}$ & 1.6 \\
\hline & G-12 & 10.3 & 5.9 & Oblong & Obtuse & $\begin{array}{c}\text { Shortly } \\
\text { Attenuate }\end{array}$ & Undulate & Sparse & Sparse & $\begin{array}{c}\text { Sparsely } \\
\text { Puberulent }\end{array}$ & 2.0 \\
\hline & G-13 & 13.4 & 5.8 & $\begin{array}{l}\text { Narrowly } \\
\text { Elliptic }\end{array}$ & Acute & Cuneate & Undulate & Sparse & Sparse & $\begin{array}{c}\text { Sparsely } \\
\text { Puberulent }\end{array}$ & 1.9 \\
\hline & G-14 & 13.2 & 7.6 & Oblong & Obtuse & Cuneate & Undulate & Sparse & Sparse & $\begin{array}{c}\text { Sparsely } \\
\text { Puberulent }\end{array}$ & 2.7 \\
\hline & G-15 & 13.4 & 5.8 & $\begin{array}{l}\text { Narrowly } \\
\text { Elliptic }\end{array}$ & $\begin{array}{l}\text { Acumi } \\
\text { nate }\end{array}$ & Cuneate & Undulate & Sparse & Sparse & $\begin{array}{c}\text { Sparsely } \\
\text { Puberulent }\end{array}$ & 2.2 \\
\hline & G-16 & 10.9 & 4.8 & $\begin{array}{l}\text { Narrowly } \\
\text { Elliptic }\end{array}$ & Acute & Cuneate & Entire & Glabrous & Glabrous & Glabrous & 1.3 \\
\hline & $\mathrm{G}-17$ & 10.9 & 6.3 & Oblong & Obtuse & $\begin{array}{c}\text { Shortly } \\
\text { Attenuate }\end{array}$ & Undulate & Sparse & Sparse & $\begin{array}{c}\text { Sparsely } \\
\text { Puberulent }\end{array}$ & 2.0 \\
\hline & G-18 & 11.4 & 6.3 & Elliptic & $\begin{array}{l}\text { Acumi } \\
\text { nate }\end{array}$ & $\begin{array}{c}\text { Shortly } \\
\text { Attenuate }\end{array}$ & Undulate & Sparse & Sparse & $\begin{array}{c}\text { Sparsely } \\
\text { Puberulent }\end{array}$ & 1.9 \\
\hline & G-19 & 9.7 & 4.9 & Elliptic & $\begin{array}{l}\text { Acumi } \\
\text { nate }\end{array}$ & Cuneate & Undulate & Sparse & Sparse & $\begin{array}{c}\text { Sparsely } \\
\text { Puberulent }\end{array}$ & 2.2 \\
\hline & $G-20$ & 8.8 & 4.1 & $\begin{array}{l}\text { Narrowly } \\
\text { Elliptic }\end{array}$ & Acute & Cuneate & Entire & Glabrous & Glabrous & Glabrous & 1.3 \\
\hline & $\begin{array}{l}\text { rand } \\
\text { Mean }\end{array}$ & 10.6 & 5.7 & & & & & & & & 1.7 \\
\hline \multirow{2}{*}{ 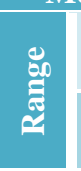 } & Max & $\begin{array}{c}13.4 \text { (G- } \\
13)\end{array}$ & $\begin{array}{c}7.6(\mathrm{G}- \\
14)\end{array}$ & & & & & & & & $\begin{array}{c}2.7 \text { (G- } \\
14)\end{array}$ \\
\hline & Min & $\begin{array}{c}7.5(\mathrm{G}- \\
10)\end{array}$ & $\begin{array}{l}4.1(\mathrm{G}- \\
20)\end{array}$ & & & & & & & & $\begin{array}{c}0.7 \text { (G- } \\
5)\end{array}$ \\
\hline
\end{tabular}

Average of 5 leaves from each genotype 
Table.3 Eigen value and variability of plant and leaf characters of jackfruit based on principal component analysis

\begin{tabular}{|c|c|c|c|c|c|c|}
\hline COMIPONENT & PC1 & PC2 & PC3 & PC4 & PC5 \\
\hline Eigen value & 5.197 & 2.565 & 2.367 & 1.535 & 1.535 \\
\hline \% Variance & 32.481 & 16.033 & 14.793 & 9.594 & 6.837 \\
\hline CHARA CTERS & \multicolumn{4}{|c|}{ EIGEN VECTORS } \\
\hline Plant height (m) & -.822 & .383 & .306 & -.013 & -.016 \\
\hline Trunk circumference (cm) & -.648 & .326 & .471 & .114 & -.175 \\
\hline Crown diameter (m) & -.584 & .615 & .348 & -.046 & -.040 \\
\hline Plant habit & -.288 & -.188 & .094 & -.673 & .460 \\
\hline Trunk surface & -.381 & -.090 & .470 & .388 & .597 \\
\hline Crown shape & .556 & -.455 & .085 & .187 & -.216 \\
\hline Leaf blade length (cm) & .205 & .500 & -.735 & -.029 & .120 \\
\hline Leaf blade width (cm) & .268 & .716 & -.295 & .172 & .209 \\
\hline Leaf blade shape & -.153 & .661 & .179 & -.427 & -.303 \\
\hline Leaf apex shape & .660 & .085 & .210 & -.106 & -.402 \\
\hline Leaf base shape & .182 & .258 & .317 & .691 & -.101 \\
\hline Leaf blade margin & .763 & .096 & .473 & -.077 & .187 \\
\hline Leaf upper surface pubescence & .782 & .271 & .270 & -.344 & .030 \\
\hline Leaf lower surface pubescence & .846 & .204 & .268 & .090 & .131 \\
\hline Leaf midrib pubescence & .793 & .202 & .430 & -.097 & .205 \\
\hline Petiole length (cm) & .276 & .493 & -.580 & .195 & .176 \\
\hline
\end{tabular}

Having medium plant height $(7.5 \mathrm{~m}), 18$ fruits/plant/season with average weight of $10 \mathrm{~kg} /$ fruit and good fruit quality characters of genotype G-13 gives an opportunity to grow as homestead plant. Easy handling small size fruit character (3kg average fruit weight) G-5 provides consumer acceptance and with the character of bearing highest number of fruit per plant among the genotypes fetching more income to farmer.

G-3, G-7 and G-13 having fruit weight greater than $10 \mathrm{~kg}$ with high TSS are highly beneficial for industries, street vendors and for home level processing units.

The result of the PCA for plant and leaf parameters of jackfruit showed the genetic diversity of the genotypes. Among the five principle component PC-1 accounted the highest variability, PC-2 and PC-3 accounted for higher variability for most of the variability observed among the jackfruit genotypes from different locations (Table 1 and Fig. 1). Among the five principal components, the first principal component axes had Eigen value > 5, the second and third principle component axes had Eigen value $>2$ and the remaining principle components had Eigen value > 1.5. PC-1, PC2, PC-3, PC-4 and PC-5 accounted for $32.48 \%, 16.03 \%, 14.79 \%, 9.59 \%$ and $6.83 \%$ respectively of the morphological variation in the jackfruit genotypes (Table 3). PC-1 explains for maximum percentage of variation and comprised of 10 characters with positive value and six characters with negative value.

\section{References}

Brown, W.H. 1941. Useful Plants of the Philippines. Dept. Agric. Natl. Res. 
Tech. Bull. Manila: Bureau of Printing, $1: 10$.

Chandan, S.M. 2001. Studies on genetic resources of jackfruit (Artocarpus heterophyllus Lamk.) in West Bengal. Ph.D. Thesis. Bidhan Chandra Krishi Viswavidyalaya, Mohanpur, West Bengal.

Chandler, W.H. 1958. Evergreen Orchards. $2^{\text {nd }}$ Ed. Henry Kimpton, London.

Chandrasekhar, V. 2014. Survey and characterization of jackfruit (Artocarpus heterophyllus Lam.) germplasm available in Andhra Pradesh to identify elite trees. Ph.D. Thesis. Dr. Y.S.R. Horticultural Venkataramannagudem, Andhra Pradesh.

Gaithoiliu, P, Lolly, S.P. and Binu, M. 2017. Diversity of jackfruit (Artocarpus heterophyllus Lam.) in Rongram Block of West Garo Hills, Meghalaya. Int. J. Sci. Env. Tech., 6(3): 1940-47.

Jager, M. I., Jarethojones, D. and Griffith, E. (1983). Component of partial resistance of wheat seedlings to Septoria nodorum.
Euphytica, 32: 575-584.

Priya, D.S., Sunetra, T., Mathala, J.G., Thangam, M. and Singh, N.P. 2014. A guide to jackfruit- cultivation and value addition. Technical bulletin No. 41, ICAR (RC), Goa. pp. 2.

Ruby, K., Nyree, Z., Salma, H. and Zuberi, M.I. 2010. Jackfruit (Artocarpus heterophyllus Lam.) Diversity in Bangladesh: Land Use and Artificial Selection. Economic Botany, 64(2): 124-36.

Terminal, W. 2012. Morphological and molecular assessment of variations clonal and seedling progenies of jackfruit (Artocarpus heterophyllus Lam.). M.Sc. Thesis. University of Agricultural Sceinces, Bangalore, Karnataka.

Wangchu, L. 2005. Selection of superior jackfruit genotypes and their identification through electrophoretic banding patterns of isozymes. Ph.D. Thesis. Bidhan Chandra Krishi Viswavidyalaya, Mohanpur, West Bengal.

\section{How to cite this article:}

Pebbuli Avani and Bauri, F.K. 2018. Morphological Variations among Different Jackfruit Genotypes. Int.J.Curr.Microbiol.App.Sci. 7(11): 3042-3048.

doi: https://doi.org/10.20546/ijcmas.2018.711.349 